\title{
Aprendizaje basado en proyectos en la obtención de papel reciclado en la asignatura de Materiales Ecoeficientes
}

José Miguel Ferria ${ }^{*}$, Harrison De La Rosa-Ramírez ${ }^{\mathrm{a}}$, Marina Patricia Arrieta ${ }^{\mathrm{b}}$, María Dolores Sampera , Juan López-Martínez ${ }^{\text {a }}$

anstituto de Tecnología de Materiales (ITM), Universitat Politècnica de València (UPV), Plaza Ferrándiz y Carbonell 1, 03801 Alcoy, Alicante, Spain.

bDepartamento de Ingeniería Química Industrial y del Medio Ambiente, Escuela Técnica Superior de Ingenieros Industriales, Universidad Politécnica de Madrid (ETSII-UPM), Calle José Gutiérrez Abascal 2, 28006 Madrid, Spain. *Corresponding author. E-mail addresses: joferaz@upvnet.upv.es (J.M. Ferri), hardela@epsa.upv.es (H. De La RosaRamírez),m.arrieta@upm.es (M.P.Arrieta),masammad@upvnet.upv.es (M. D. Samper), jlopezm@mcm.upv.es (J. López-Martínez).

\section{\$EWWFW}

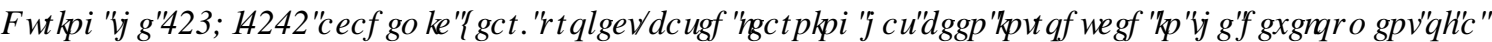

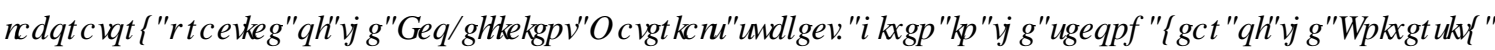

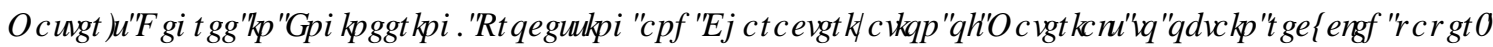

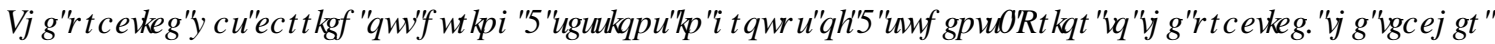

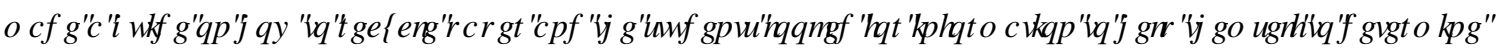

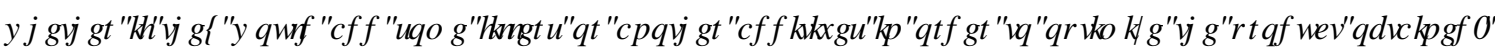

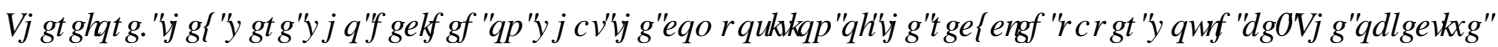

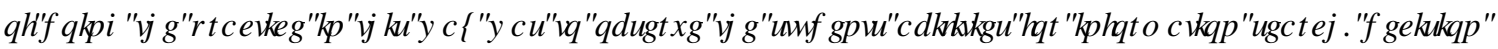

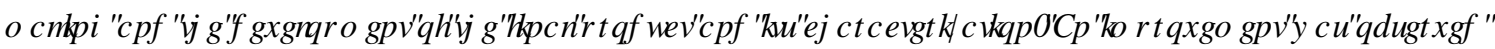

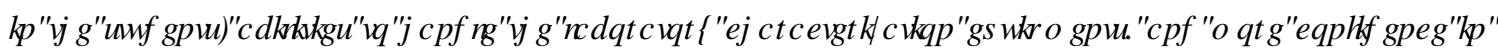
$P$ DNQJ $L G F L M R Q W$

Keywords: methodology, project-based learning, teaching innovation, laboratory practices.

\section{HXP HQD}

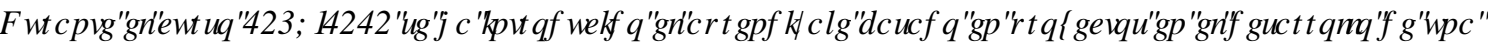

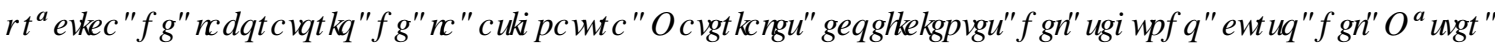

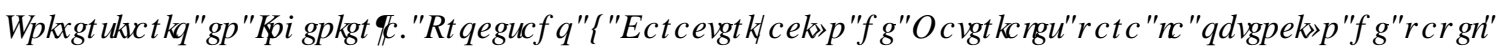

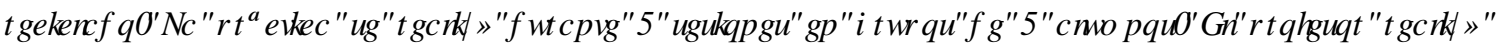

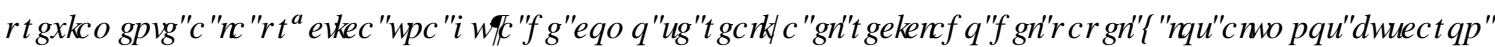

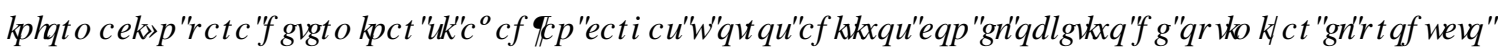

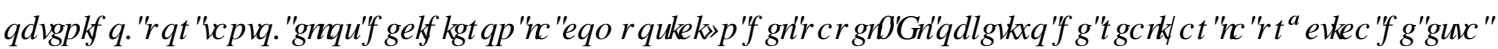

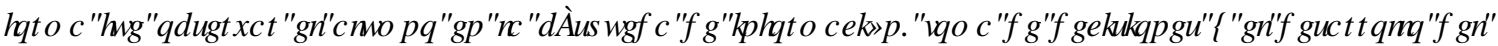

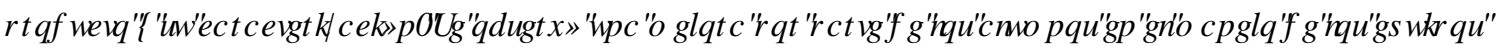

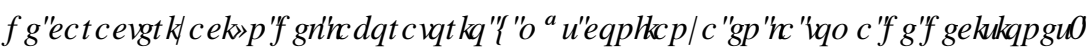

Palabras clave: metodología, aprendizaje basado en proyectos, innovación docente, prácticas de laboratorio. [ 


\section{Introducción}

Cada vez es más habitual el uso de métodos de enseñanza donde el alumno desempeña un papel activo en el proceso enseñanza-aprendizaje y que le ayuda a tener un mejor pensamiento crítico. Durante diversos estudios se ha demostrado que los alumnos retienen más conocimiento en experiencias prácticas y discusión en grupos, cuando ellos son participativos, que en clases magistrales, donde ellos son sujetos pasivos (Rodríguez-Sandoval \& Luna-Cortés, 2010)

Se ha demostrado que para conseguir el desarrollo de competencias, una de las principales medidas del Espacio Europeo de Educación Superior, es mucho más viable el uso de métodos docentes donde el alumno es el protagonista en el proceso de enseñanza-aprendizaje y el profesor pasa a tener un rol secundario como guía en este proceso. Una de las metodologías que fomenta este cambio, respecto a metodologías tradicionales, es el Aprendizaje Basado en Proyectos (ABP o ABPr) incluidas dentro de las "metodologías activas". Alguno de los puntos fundamentales del ABP es que los alumnos se sitúan en un contexto real, están implicados en el diseño del proyecto, resuelven dudas y toman decisiones sobre su ejecución (Ausín \& Abella, 2016).

En la metodología ABP el aprendizaje del alumno es el resultado de su esfuerzo al realizar un proyecto. Este proyecto se suele realizar en grupos y deben realizar (Ruiz et al., 2003).

- Identificar los conocimientos previos del grupo y lo que deben aprender para realizar el proyecto con éxito.

- Establecer y llevar a cabo un plan de aprendizaje.

- Revisar el proyecto en base al aprendizaje que han adquirido y determinar si con lo que han aprendido se podría enfocar de otra forma.

El rol del profesor es secundario y actúa como supervisor, aunque puede impartir o sugerir algún conocimiento necesario y básicamente debe de realizar las siguientes tareas (Ruiz et al., 2003).

- Formular proyectos interesantes

- Facilitar el plan de aprendizaje de cada grupo

- Ofrecer "feedback" a cada grupo sobre el desarrollo del proyecto

- Guiarles en la resolución de problemas

Para conseguir que los alumnos sean los protagonistan en el proceso enseñanza-aprendizaje es necesario una madurez y compromiso activo por su parte, y es más exitoso si se desarrolla en últimos cursos y en grupos reducidos.

Entre las capacidades que pueden desarrollar los alumnos con esta metodología destacan el trabajo en equipo, el aprendizaje autónomo, el pensamiento crítico, la planificación del tiempo y la capacidad de expresión oral y escrita (Reverte Bernabeu et al., 2007). Además, mejora la motivación en alumnos y el interés por la asignatura, $\mathrm{y}$, por tanto, un mayor rendimiento, tanto a nivel académico como personal.

En este trabajo se propone el uso de la metodología ABP en el desarrollo de una práctica de laboratorio sobre el reciclado de papel de la asignatura "Materiales Ecoeficientes" impartida en el segundo curso del Máster Universitario en Ingeniería, Procesado y Caracterización de Materiales impartido en la Escuela Politécnica Superior de Alcoy (UPV-campus Alcoy) con un total de 9 alumnos matriculados durante el curso 2019/2020. Se ha optado por esta metodología para que los alumnos adquieran un rol activo y desarrollen una cultura de trabajo en equipo y toma de decisiones con una planificación detallada en el tiempo, de forma similar a como se desarrolla en el ámbito laboral. Además, fomenta el autoaprendizaje y la interacción entre los alumnos para el desarrollo de nuevas habilidades y sintiéndose responsables de los resultados obtenidos (Hass, 2000)(Pavon et al., 2019). El fin de dicho trabajo es autoevaluar la metodología 
aplicada por el docente a través de una encuesta realizada por los propios alumnos indicando como resultado el índice de aceptación de ésta.

Para la realización del proyecto, se facilitó a los alumnos una guía sobre la metodología para obtener papel reciclado y se dividió selectivamente a éstos en grupos de 3 personas. El proyecto consistía en la mejora u optimización de papel reciclado añadiéndole cargas/aditivos naturales y los alumnos buscaron información sobre la temática planteada y decidieron los productos naturales a utilizar, así como su tipología y cantidad. Se consiguió fomentar la autonomía de los alumnos, ya que fueron los protagonistas en la toma de decisiones, quedando el profesor en un segundo plano.

\section{Objetivos}

- Fomentar el trabajo en equipo y la toma de decisiones de los alumnos mediante el uso de la metodología deABP para el desarrollo de una práctica de laboratorio en la asignatura de "Materiales Ecoeficientes" de segundo curso del Master Universitario en Ingeniería, Procesado y Caracterización de Materiales".

- Simular un entorno laboral sobre el proceso productivo empleado habitualmente para la fabricación del papel, ya que son alumnos de último curso de máster.

- Evaluar el nivel de implicación de alumnado a la hora de tomar decisiones, de llevarlas a cabo y del uso del carácter crítico a la hora de resolver problemas.

\section{Desarrollo de la innovación}

A continuación se explican las pautas tenidas en cuenta para el empleo de la metodología "ABP" aplicada a la asignatura de "Materiales Ecoeficientes". La implementación de dicha metodología necesita meditarse por parte del docente. Con ello se pretende evaluar el grado de seguridad, autoestima, poder de resolución de problemas adquirido por parte del alumno y evaluar el grado de satisfacción basado en el aprendizaje de los alumnos con la implementación de dicha metodología.

\section{- Preparación de las clases:}

Previamente a la práctica el alumnado ya contaba con una guía de ésta donde se indicaban los pasos para producir papel reciclado. Los alumnos disponían de todo el material y utensilios necesarios para la realización del papel. Los objetivos específicos de la práctica fueron el dar respuesta con evidencias a la siguiente pregunta formulada por el docente.

\section{Ф( VSRUEGIDXP HQWUOUUMWUDFIDI [FRHIFIHQFIDGHXQSDSHDUFIFOGR"]}

Cada grupo, formado por 3 alumnos, planificó, bajo la sugerencia del docente, el trabajo de cada sesión de la práctica y las tareas a realizar por cada uno de sus componentes para que en las tres sesiones ( 2 horas cada una) tuvieran tiempo a planificar los trabajos, decidir las pruebas de papel a realizar, caracterizarlo, analizar los resultados y exponerlos en la última sesión de la práctica. El tiempo programado por el docente fue medido a conciencia para que el alumnado no dispusiera de tiempo sobrante con el objetivo de añadir presión. El objetivo fue el de simular un ambiente laboral real.

Para que el alumnado pudiera hacerse una idea de los objetivos a conseguir el docente realizó una rúbrica de evaluación de la práctica (Tabla 1). 


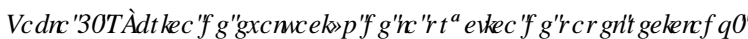

Rúbrica para evaluar la práctica de papel reciclado

\begin{tabular}{|c|c|c|c|c|c|}
\hline $\begin{array}{c}\% \\
\max \end{array}$ & INDICADORES & Insuficiente & Suficiente & Notable & Excelente \\
\hline 16 & Planificación & $\begin{array}{l}\text { La planificación } \\
\text { realizada no es } \\
\text { lo } \\
\text { suficientemente } \\
\text { buena para } \\
\text { realizar la } \\
\text { práctica a } \\
\text { tiempo.(4\%) }\end{array}$ & $\begin{array}{c}\text { La planificación } \\
\text { realizada permite } \\
\text { que se terminen } \\
\text { todas las etapas } \\
\text { dentro del tiempo } \\
\text { establecido aunque } \\
\text { no se distribuyen las } \\
\text { tareas de forma } \\
\text { equitativa.(8\%) }\end{array}$ & $\begin{array}{l}\text { La planificación } \\
\text { realizada permite } \\
\text { que se terminen } \\
\text { todas las etapas } \\
\text { dentro del tiempo } \\
\text { establecido y se } \\
\text { distribuyen las } \\
\text { tareas de forma } \\
\text { equitativa.(12\%) }\end{array}$ & $\begin{array}{l}\text { La planificación } \\
\text { realizada permite que } \\
\text { se terminen todas las } \\
\text { etapas dentro del } \\
\text { tiempo establecido y } \\
\text { se distribuyen las } \\
\text { tareas de forma } \\
\text { optimizada. }(16 \%)\end{array}$ \\
\hline 34 & Resultados & $\begin{array}{l}\text { No se han } \\
\text { obtenido } \\
\text { suficientes } \\
\text { resultados para } \\
\text { obtener } \\
\text { conclusiones. } \\
(8,5 \%)\end{array}$ & $\begin{array}{c}\text { Se obtienen } \\
\text { resultados } \\
\text { coherentes con los } \\
\text { objetivos aunque sin } \\
\text { aportar evaluaciones } \\
\text { y justificaciones de } \\
\text { dichos resultados. } \\
(17 \%)\end{array}$ & $\begin{array}{c}\text { Se obtienen } \\
\text { resultados } \\
\text { coherentes con los } \\
\text { objetivos y además } \\
\text { evaluaciones y } \\
\text { justificaciones de } \\
\text { dichos resultados. } \\
(25,5 \%)\end{array}$ & $\begin{array}{l}\text { No solo se obtienen } \\
\text { resultados coherentes, } \\
\text { sino que se justifican } \\
\text { con la literatura y se } \\
\text { entiende y presenta } \\
\text { de forma muy clara y } \\
\text { rigurosa. }(34 \%)\end{array}$ \\
\hline 28 & Exposición & $\begin{array}{l}\text { La exposición } \\
\text { no contiene } \\
\text { todas las fases } \\
\text { de la práctica } \\
\text { por lo que no } \\
\text { pueden alcanzar } \\
\text { el objetivo o no } \\
\text { se expone con } \\
\text { claridad. (7\%) }\end{array}$ & $\begin{array}{c}\text { La exposición } \\
\text { contiene todas las } \\
\text { fases de la práctica } \\
\text { y se expone con } \\
\text { claridad aunque no } \\
\text { muestra evidencias } \\
\text { bien justificadas, ya } \\
\text { sean por resultados } \\
\text { positivos o } \\
\text { negativos. }(14 \%)\end{array}$ & $\begin{array}{c}\text { La exposición } \\
\text { contiene todas las } \\
\text { fases de la práctica } \\
\text { y se expone con } \\
\text { claridad mostrando } \\
\text { evidencias bien } \\
\text { justificadas, ya sean } \\
\text { por resultados } \\
\text { positivos o } \\
\text { negativos. }(21 \%)\end{array}$ & $\begin{array}{l}\text { La exposición es } \\
\text { completa, muy clara, } \\
\text { bien justificada, } \\
\text { muestra un apartado } \\
\text { de tareas a realizar } \\
\text { para mejorar los } \\
\text { resultados obtenidos } \\
\text { que indica que los } \\
\text { alumnos han } \\
\text { investigado como } \\
\text { mejorar las } \\
\text { propiedades del } \\
\text { papel. }(28 \%)\end{array}$ \\
\hline 22 & $\begin{array}{l}\text { Carácter crítico y } \\
\text { trabajo en equipo }\end{array}$ & $\begin{array}{l}\text { No lo aplican. } \\
\qquad(5,5 \%)\end{array}$ & $\begin{array}{l}\text { Lo aplican aunque } \\
\text { no muestran } \\
\text { seguridad. (11\%) }\end{array}$ & $\begin{array}{c}\text { Se aplica un buen } \\
\text { carácter crítico y } \\
\text { bien fundamentado. } \\
(16,5 \%)\end{array}$ & $\begin{array}{l}\text { Se aplica de forma } \\
\text { bien fundamentada y } \\
\text { se muestran muy } \\
\text { seguros al defender } \\
\text { sus resultados. }(22 \%)\end{array}$ \\
\hline
\end{tabular}

El docente sabe que el alumnado ya conoce las técnicas de caracterización suficientes para poder evaluar los resultados y evaluará el buen criterio del alumnado a la hora de seleccionar dichas técnicas de caracterización. Además, el profesor actúa en todo momento como supervisor por si detecta problemas importantes durante la caracterización.

En la Tabla 2 se observa un resumen de la consecución de las prácticas desde la fabricación del papel, pasando por la caracterización y un análisis final. 


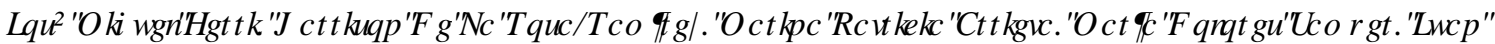
/ ySH $\square 0$ DUWQH]

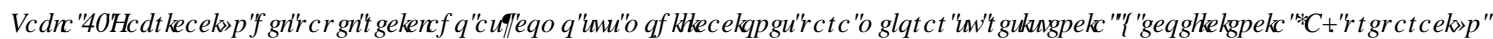

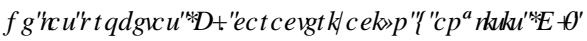

A
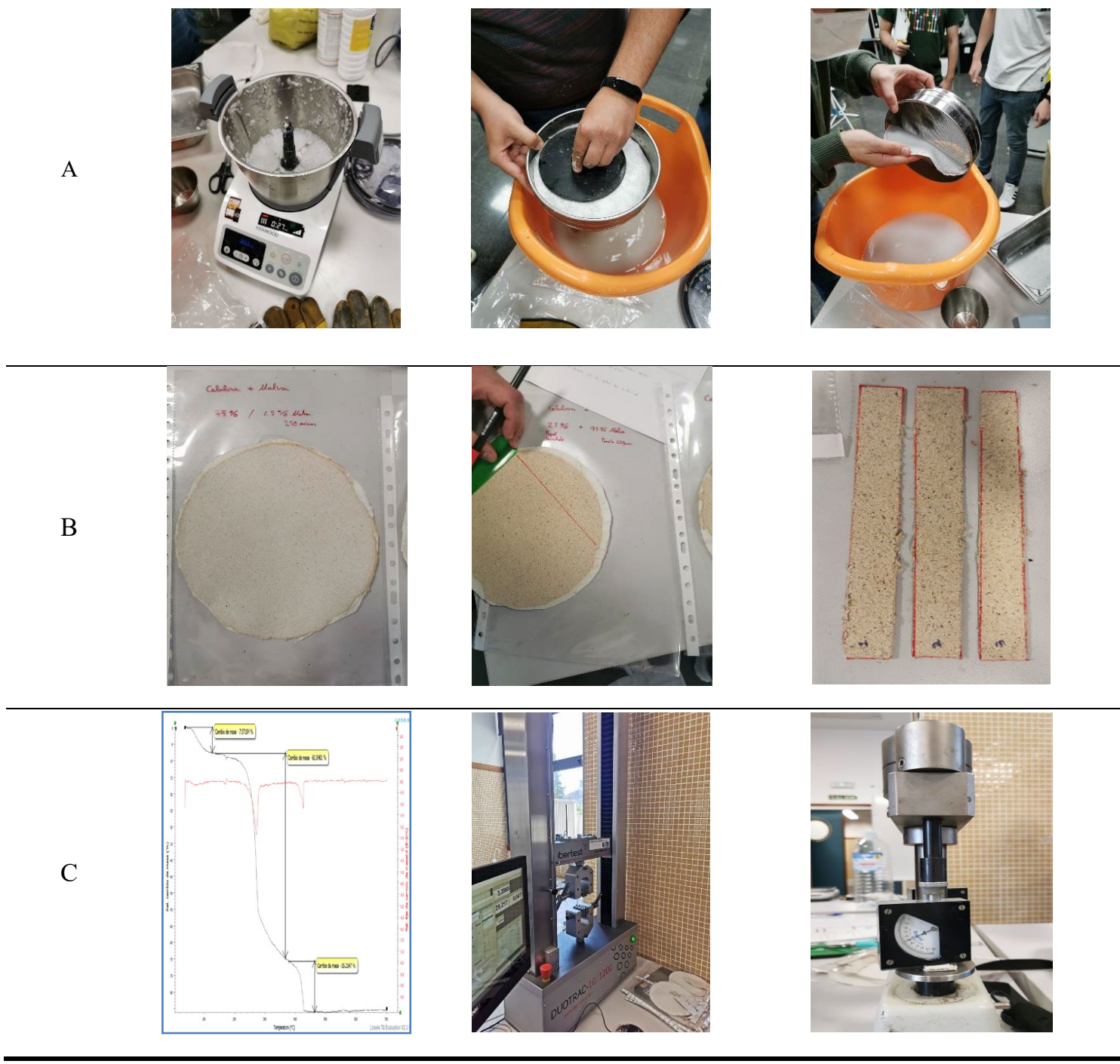

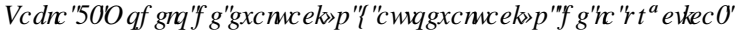

Evaluación de la práctica de papel reciclado mediante rúbrica

\begin{tabular}{|c|c|c|c|c|c|c|c|c|c|}
\hline $\begin{array}{c}\% \\
\max \end{array}$ & ALUMNOS & 1 & 2 & 3 & 4 & 5 & 6 & 7 & 8 \\
\hline 16 & Planificación & & & & & & & & \\
\hline 34 & Resultados & & & & & & & & \\
\hline 28 & Exposición & & & & & & & & \\
\hline 22 & $\begin{array}{l}\text { Carácter crítico y } \\
\text { trabajo en equipo }\end{array}$ & & & & & & & & \\
\hline
\end{tabular}

TOTAL

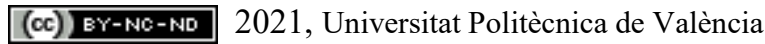

CRQJUHRL, Q5 HGHपिए। 
Una vez realizada la práctica el docente realizó una evaluación a los alumnos como la que se muestra en la Tabla 3 y los alumnos completaron la misma tabla como ejercicio de autoevaluación para observar su carácter reflexivo y autocrítica siguiendo la rúbrica aportada a éstos.

Finalmente, los alumnos evaluaron la experiencia de la metodología aplicada para observar el nivel de aceptación del ABP mediante la plataforma GOOGLE FORMS (Figura 2).

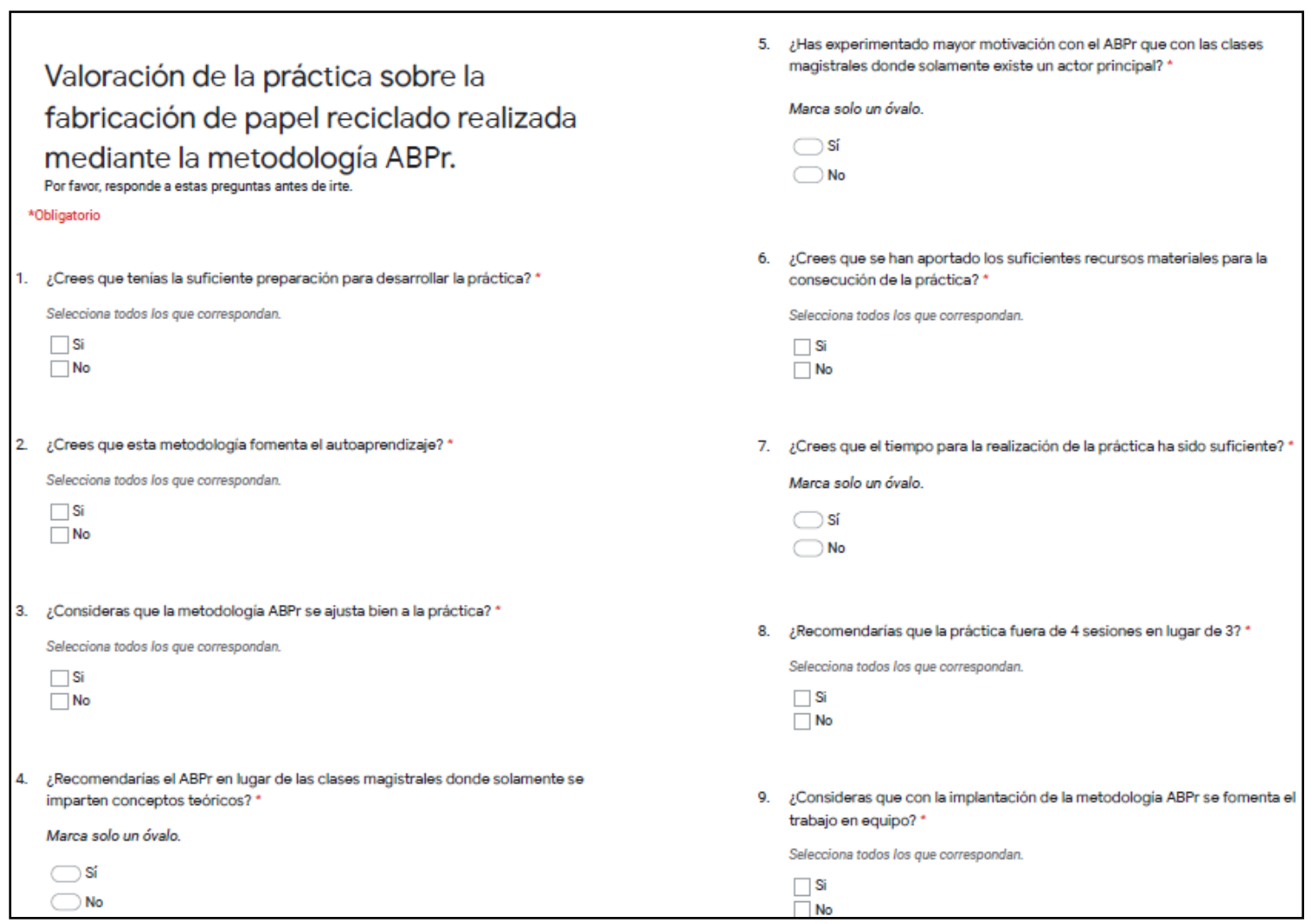

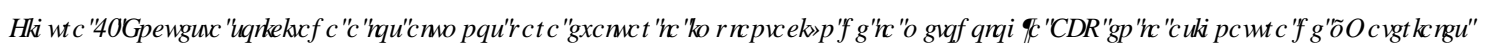

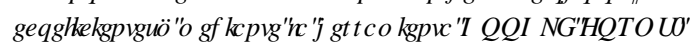

\section{Resultados}

En la asignatura "Materiales ecoeficientes" impartida mediante la metodología de ABP participaron 9 de los 9 alumnos matriculados. La participación activa de los alumnos fue del 100\% gracias a que fue un grupo muy reducido y la propia metodología, junto con el tiempo ajustado para la realización de la práctica, obligó a que todos los alumnos participasen en la consecución de la fabricación, caracterización y análisis de la fabricación de papel ecoeficiente. Hay que tener en cuenta que con esta metodología son los propios alumnos los que motivan a sus compañeros de grupo a desarrollar el proyecto como única opción para conseguir el objetivo de la práctica. Una vez expuestos los resultados de la práctica, se planteó a los alumnos realizar de forma anónima una evaluación de la metodología empleada en la asignatura, mediante la plataforma GOOGLE FORMS. Para ello se utilizó un cuestionario realizado por el docente y se envio por correo electrónico a todos los alumnos. En la Tabla 4 se resumen estos resultados. Se observa que al menos dos terceras partes de la clase estaban suficientemente preparados para desarrollar la práctica. La metodología permite que esas dos terceras partes que sí estan preparadas puedan ayudar a la otra parte a 


\section{/ ySH $\square 0$ DURQ $]$}

desarrollar la práctica, porque se trata de un trabajo en grupo donde los alumnos más preparados pueden ayudar a los que no lo están.

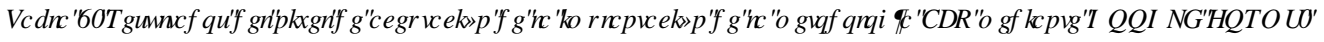

Evaluación de la práctica de papel mediante GOOGLE FORMS.

\begin{tabular}{|c|c|c|c|c|c|c|c|c|c|}
\hline PREGUNTA & 1 & 2 & 3 & 4 & 5 & 6 & 7 & 8 & 9 \\
\hline $\begin{array}{c}\text { ¿Crees que tenías la suficiente preparación para } \\
\text { desarrollar la práctica? }\end{array}$ & SÍ & SÍ & SÍ & $\mathrm{NO}$ & $\mathrm{NO}$ & SÍ & SÍ & $\mathrm{NO}$ & SÍ \\
\hline $\begin{array}{c}\text { ¿Crees que esta metodología fomenta el } \\
\text { autoaprendizaje? }\end{array}$ & SÍ & SÍ & SÍ & SÍ & SÍ & SÍ & SÍ & SÍ & SÍ \\
\hline $\begin{array}{c}\text { ¿Consideras que la metodología ABP se ajusta bien a } \\
\text { la práctica? }\end{array}$ & SÍ & SÍ & SÍ & SÍ & $\mathrm{NO}$ & SÍ & SÍ & SÍ & SÍ \\
\hline $\begin{array}{c}\text { ¿Recomendarías el ABP en lugar de las clases } \\
\text { magistrales donde solamente se imparten conceptos } \\
\text { teóricos? }\end{array}$ & SÍ & SÍ & Sí & SÍ & $\mathrm{NO}$ & SÍ & SÍ & SÍ & SÍ \\
\hline $\begin{array}{l}\text { ¿Has experimentado mayor motivación con el ABP } \\
\text { que con las clases magistrales donde solamente existe } \\
\text { un actor principal? }\end{array}$ & SÍ & SÍ & SÍ & SÍ & NO & SÍ & SÍ & SÍ & SÍ \\
\hline $\begin{array}{l}\text { ¿Crees que se han aportado los suficientes recursos } \\
\text { materiales para la consecución de la práctica? }\end{array}$ & SÍ & SÍ & SÍ & SÍ & $\mathrm{NO}$ & SÍ & SÍ & SÍ & SÍ \\
\hline $\begin{array}{l}\text { ¿Crees que el tiempo para la realización de la práctica } \\
\text { ha sido suficiente? }\end{array}$ & $\mathrm{NO}$ & SÍ & $\mathrm{NO}$ & SÍ & SÍ & $\mathrm{NO}$ & $\mathrm{NO}$ & $\mathrm{NO}$ & $\mathrm{NO}$ \\
\hline $\begin{array}{c}\text { ¿Recomendarías que la práctica fuera de } 4 \text { sesiones en } \\
\text { lugar de } 3 \text { ? }\end{array}$ & Sí & $\mathrm{NO}$ & SÍ & $\mathrm{NO}$ & $\mathrm{NO}$ & SÍ & SÍ & SÍ & SÍ \\
\hline
\end{tabular}

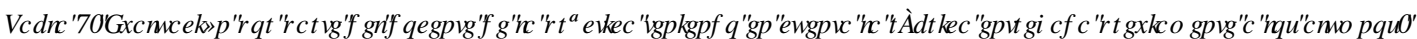

Evaluación de la práctica de papel reciclado mediante rúbrica

\begin{tabular}{ccccccccccc}
\hline $\begin{array}{c}\% \\
\max \end{array}$ & ALUMNO & 1 & 2 & 3 & 4 & 5 & 6 & 7 & 8 & 9 \\
\hline 16 & Planificación & 12 & 12 & 12 & 12 & 16 & 16 & 16 & 16 & 12 \\
\hline 34 & Resultados & 25,5 & 25,5 & 25,5 & 25,5 & 34 & 34 & 34 & 34 & 25,5 \\
\hline 28 & Exposición & 21 & 28 & 28 & 21 & 28 & 21 & 14 & 21 & 14 \\
\hline 22 & $\begin{array}{c}\text { Carácter crítico y } \\
\text { trabajo en equipo }\end{array}$ & 16,5 & 22 & 22 & 16,5 & 16,5 & 16,5 & 11 & 22 & 22 \\
\hline & TOTAL & 75 & 87,5 & 87,5 & 75 & 94,5 & 87,5 & 75 & 93 & 73,5 \\
\hline
\end{tabular}

El 100\% de los alumnos consideró que el método fomenta el autoaprendizaje, objetivo que se persigue con la implantación de dicha metodología. Se debe tener en cuenta que los alumnos deben estar capacitados para aprender por su cuenta puesto que cuando acaben el curso ingresarán en el mundo laboral. Un 88,9\% de los alumnos ( 8 de 9 ) consideró que la asignatura podía implantar la metodología ABP y concretamente 
para la práctica realizada. El mismo porcentaje de alumnos consideró que la metodología ABP es mejor para impartir la asignatura que las clases magistrales y que experimentaron mayor motivación con dicha metodología. Sin embargo, el 66,6\% de los alumnos consideró que el tiempo para desarrollar la práctica adecuadamente era insuficiente y consideraron que sería necesaria otra sesión adicional. Puesto que uno de los objetivos era el de simular un ambiente laboral, se consideró lógico el resultado de las dos últimas preguntas ya que todos los alumnos no responden de igual forma bajo situaciones de presión.

Por otra parte, el trabajo desarrollado durante la práctica ha sido muy positivo y se refleja en las puntuaciones aplicadas por el docente (Tabla 5). La mayor implicación y motivación del alumnado han sido las principales razones. La calificación media obtenida de la práctica en el curso 2019/2020 fue de 8,3 $\pm 0,9$ puntos sobre 10 . Dicha calificación se consideró alta aunque lógica por la alta implicación del alumnado.

Finalmente el profesor quiso identificar el grado de autocrítica y de reflexión por parte de los alumnos con los datos de la autoevaluación. Seis de los nueve alumnos se autoevaluaron con cualificaciones inferiores a las asignadas por el docente (en rojo en la Tabla 6) y tres se evaluaron correctamente con respecto a los criterios de la rúbrica. Esto significa que la gran mayoría asumió que se podía mejorar en algunos de los aspectos evaluados en la práctica.

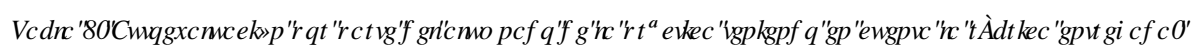

\begin{tabular}{ccccccccccc}
\hline \multicolumn{7}{c}{ Autoevaluación de la práctica de papel reciclado mediante rúbrica } \\
\hline $\begin{array}{c}\text { max } \\
\text { ALUMNO }\end{array}$ & 1 & 2 & 3 & 4 & 5 & 6 & 7 & 8 & 9 \\
\hline 16 & Planificación & 12 & 12 & 12 & 12 & 16 & 12 & 12 & 16 & 12 \\
\hline 34 & Resultados & 17 & 25,5 & 17 & 25,5 & 34 & 25,5 & 25,5 & 25,5 & 17 \\
\hline 28 & Exposición & 21 & 28 & 21 & 21 & 28 & 21 & 21 & 21 & 14 \\
\hline 22 & $\begin{array}{c}\text { Carácter crítico } \\
\text { y trabajo en } \\
\text { equipo }\end{array}$ & 16,5 & 22 & 16,5 & 16,5 & 16,5 & 16,5 & 11 & 22 & 22 \\
\hline
\end{tabular}

\section{Conclusiones}

La aplicación de la metodología ABP en la asignatura "Materiales ecoeficientes" ha demostrado ser una muy buena experiencia para los alumnos y para el docente, conclusión a la que se llega a partir de las cualificaciones obtenidas de la práctica y de los resultados de la encuesta de valoración realizada por los alumnos sobre la metodología. Se observa una alta aceptación por parte de los alumnos, según dicha encuesta anónima donde la gran mayoría reconoce haber estado mas motivado que en otras metodologías, como es el caso de las clases magistrales.

De la implantación de la metodología ABP se destaca un claro autoaprendizaje por parte de los alumnos y una alta colaboración entre los componentes de los grupos. En lo que no hay consenso es en el tiempo dedicado a la práctica, aunque era previsible dado que no todos los alumnos tienen las mismas capacidades ante situaciones de presión que simulan un entorno real de trabajo.

Dado que la aceptación de dicha metodología ha sido alta, se continuará aplicando ésta para seguir estudiando sus efectos en la asignatura de Materiales ecoeficientes. 


\section{Referencias}

Ausín, V., \& Abella, V. (2016). “Aprendizaje Basado en Proyectos a través de las TIC. Una Experiencia de Innovación Docente desde las Aulas Universitarias". en ) RUP DFIy Q8 QIHHWWWD, vol. 9, issue 3, p. 3138 .

Hass, M. A. (2000). "Student-Directed Learning in the Organic Chemistry Laboratory". en -RXLQDORI \&KHP IFDO GXFDURQ vol. 77, issue 8, p. 1035-1038.

Pavon, C., Aldas, M., H De La Rosa, H., \& López-Martínez, J. (2019). “Aprendizaje activo y cooperativo en el laboratorio de Nanotecnolo- gía para realizar estructuras con electrospinning / electrospraying

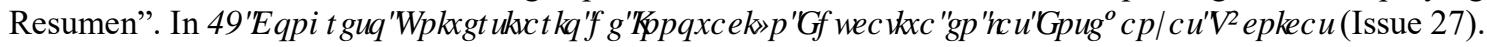

Reverte Bernabeu, J. R., Javier Gallego Sánchez, A., Molina Carmona, R., Satorre Cuerda, R., \& San Vicente del Raspeig, C. (2007). "El Aprendizaje Basado en Proyectos como modelo docente. Experiencia interdisciplinar y herramientas Groupware". en ; , , , -RLQDODV GH ( QUKDOJD 8 QIVHWWDUD GH / D

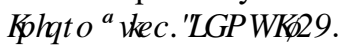

Rodríguez-Sandoval, E., \& Luna-Cortés, J. (2010). "Evaluación de la estrategia, Aprendizaje basado en proyectos". en ( GXFL( GXFL, vol. 13, issue 1, p. 13-25.

Ruiz, S., Valero, M., \& Alcober, J. (2003). "Evaluación de la implantación del aprendizaje basado en proyectos en la EPSC ( 2001-2003 )”. en \$FWD' HD; , L\&RQJ UHR 8 QIVHUWDUR] GH, QQRIDFIYQ( GXFDWMD ( QV DVK QMHDQIDV7pFQIFDV, p. 10. 